\title{
Positive Association Between Limbic Metabotropic Glutamate Receptor 5 Availability and Novelty-Seeking Temperament in Humans: An ${ }^{18}$ F-FPEB PET Study
}

\author{
Gil Leurquin-Sterk ${ }^{1}$, Jan Van den Stock ${ }^{2}$, Cleo Lina Crunelle ${ }^{3}$, Bart de Laat ${ }^{1,4}$, Akila Weerasekera ${ }^{5}$, Uwe Himmelreich ${ }^{5}$, \\ Guy Bormans ${ }^{6}$, and Koen Van Laere ${ }^{1,4}$ \\ ${ }^{I}$ Department of Nuclear Medicine and Molecular Imaging, University Hospitals Leuven, Leuven, Belgium; ${ }^{2}$ Laboratory for \\ Translational Neuropsychiatry, Department of Neurosciences, KU Leuven and Department of Old Age Psychiatry, University \\ Hospitals Leuven, Leuven, Belgium; ${ }^{3}$ Toxicological Center, University of Antwerp, Wilrijk, Belgium; ${ }^{4}$ MoSAIC, Molecular Small \\ Animal Imaging Center, KU Leuven, Leuven, Belgium; ${ }^{5}$ Biomedical MRI/MoSAIC, Department of Imaging and Pathology, \\ $K U$ Leuven, Leuven, Belgium; and ${ }^{6}$ Laboratory for Radiopharmacy, KU Leuven, Leuven, Belgium
}

\begin{abstract}
Heritable temperament traits have been linked to several neuropsychiatric illnesses, including disorders associated with metabotropic glutamate receptor 5 (mGluR5) and dopaminergic dysfunctions. Considering its modulating effect on neurotransmission, we hypothesized that cerebral mGluR5 availability is associated with temperament traits in healthy humans. Methods: Forty-four nonsmoking healthy volunteers (mean age $\pm \mathrm{SD}, 40 \pm 14$ y; age range, 22-66 y; 22 women) were included in this cross-sectional investigation. Brain mGluR5 availability was quantified on both a voxel-by-voxel and a volume-of-interest basis using the total distribution volume of the radioligand ${ }^{18} \mathrm{~F}-3-$ fluoro-5[(pyridin-3-yl)ethynyl]benzonitrile ( $\left.{ }^{18} \mathrm{~F}-\mathrm{FPEB}\right)$ with 90-min dynamic PET and arterial input function. Moreover, glutamate-glutamine concentrations in the anterior cingulate cortex were measured using MR spectroscopy. These measures were related to the temperament traits of the 240-item Cloninger temperament and character inventory using a regression analysis with age and sex as nuisance variables. Results: High novelty-seeking temperament was robustly associated with increased mGluR5 availability in various regions including the thalamus $(r=0.71$; the strongest association), amygdala, parahippocampus, insula, anterior and posterior cingulate cortex, and several primary sensory areas (all $r>$ $0.58 ; P<0.05$, corrected for familywise error). These associations were specific because no correlations were found with other temperament scales or with spectroscopic measures of glutamatergic transmission. Conclusion: Overall, these data posit mGluR5 in key paralimbic areas as a strong determinant of the temperament trait novelty seeking. These data add to our understanding of how brain neurochemistry accounts for the variation in human behavior and strongly support further research on mGluR5 as a potential therapeutic target in neuropsychiatric disorders associated with abnormal novelty-seeking behaviors.
\end{abstract}

Key Words: neuroimaging; ${ }^{18} \mathrm{~F}-\mathrm{FPEB}$; mGluR5; personality; novelty seeking

J Nucl Med 2016; 57:1746-1752

DOI: 10.2967/jnumed.116.176032

Received Mar. 21, 2016; revision accepted May 6, 2016.

For correspondence or reprints contact: Gil Leurquin-Sterk, Division of Nuclear Medicine, University Hospital Leuven, Herestraat 49, 3000 Leuven, Belgium.

E-mail: gil.leurquin-sterk@uzleuven.be

Published online Jun. 9, 2016.

COPYRIGHT (c) 2016 by the Society of Nuclear Medicine and Molecular Imaging, Inc.
$\mathbf{T}$ he neurobiologic substrates of human personality and behavior remain an important but complex area of neuroscience. More than 20 y ago, the Cloninger's theory of personality represented one of the first attempts to integrate neurotransmitter systems into personality research (1). This model identified 7 personality traits, that is, 4 temperaments and 3 characters. Temperaments are thought to be independently inherited with specific brain circuits and stable over a lifetime, whereas character dimensions are suggested to form over time through the interplay of genetic and environmental factors. Subsequently, genetic determinants of receptor expression and neuronal function in the normal variation of human behavior have been validated (2). Several temperament and personality traits, including novelty seeking (NS), harm avoidance, and impulsivity, have been associated with the vulnerability to mental illnesses $(3,4)$ and have been directly related to receptors and neurotransmission using functional imaging (5-7). In parallel, dysregulation or disequilibrium of brain neurotransmitters have repeatedly been linked to mental diseases. Therefore, a better understanding of neurotransmitter systems and their involvement in personality and behavior may subsequently pave the way for improved treatment strategies for a multitude of neuropsychiatric disorders.

Though temperament research has mainly implicated central monoaminergic systems, mounting evidence points toward a more complex neurotransmitter interplay to account for the variation in human personality $(5,6,8)$. Glutamate is the main excitatory neurotransmitter in the brain and is involved in numerous aspects of behavior and cognition. Glutamate concentrations in the anterior cingulate cortex (ACC) have been associated with personality and behavior including sensation seeking (8), harm avoidance (9), reward processing (10), anxiety (11), impulsivity, and aggression (7). On release, glutamate can bind to metabotropic glutamate receptors (mGluR) located pre-, post- and perisynaptically, which are in turn responsible for modulating the synaptic transmission of glutamate and other neurotransmitters. Hence, exploring specific components of this modulatory system is an appealing approach to refine our understanding of the biologic correlates of human behavior. mGluR5 is a Gq/11-protein-coupled receptor mainly located on postsynaptic terminals where it positively modulates the $N$-methyl-D-aspartate receptor by increasing open channel probability (12). Moreover, mGluR5-mediated retrograde endocannabinoid signaling reduces presynaptic neurotransmitter release of 
both excitatory and inhibitory terminals (13). Importantly, mGluR5 is highly expressed in limbic cortical and subcortical areas receiving dopaminergic inputs (14). In rodents, blocking mGluR5 signaling reduces anxiety (15), NS (16), and addictive behaviors (16,17). Accordingly, the mGluR5 has been proposed as an important target for the treatment of many mental disorders. During the last decade, in vivo measurement of mGluR5 availability by PET with the radioligand ${ }^{18} \mathrm{~F}$-3-fluoro-5-[(pyridin-3-yl)ethynyl]benzonitrile $\left({ }^{18} \mathrm{~F}-\mathrm{FPEB}\right)$, a selective mGluR5-negative allosteric modulator with high affinity (dissociation constant, $0.05 \mathrm{nmol} / \mathrm{L}$ ), became possible (18). However, research on the involvement of mGluR5 in human personality is lacking.

Here, we assessed the association between brain mGluR5 availability and the temperament dimensions of the Cloninger's psychobiologic theory of personality (19). Additionally, given the suggested involvement of ACC glutamate concentrations in personality and their possible influence on mGluR5 availability (20), mGluR5 and temperament measures were related to spectroscopic measures of ACC glutamate-glutamine levels.

\section{MATERIALS AND METHODS}

\section{Participants}

Forty-four male and female healthy volunteers aged 22-66 y were recruited through local newspaper and internet advertisements. These controls are also currently involved in a larger study on the involvement of mGluR5 in drug addiction. Nine of these volunteers were previously included in a study assessing the long-term test-retest reproducibility of ${ }^{18}$ F-FPEB (21); only their baseline data were used for this study. Normal physical and mental health was attested by means of medical history, clinical evaluation, routine blood and urine laboratory tests, urine drug screening (Multi Urine Drugcontrol cassette and Cotinine Card Test; Ultimed Products), neuropsychologic testing, and structural brain MR imaging. The Mini-International Neuropsychiatric Interview was used to exclude Diagnostic and Statistical Manual of Mental Disorders IV psychiatric disorders (22). Exclusion criteria were any abnormal findings on above-mentioned tests; pregnancy; breastfeeding; a history of psychiatric disorder in the subject or in a first-degree relative; current use of psychotropic drugs; and current or past history of smoking, illicit drug abuse, and alcohol consumption of more than 7 units per week. Actual excessive alcohol consumption was further excluded by the self-report Alcohol Use Disorder Identification Test (23) and by quantitative ethyl glucuronide scalp hair analysis.

The study was approved by the local Ethics Committee, and all subjects signed a written informed consent form and received a stipend.

\section{Personality Assessment}

Temperament traits were assessed using the validated Dutch version (24) of the 240-item Cloninger temperament and character inventory (TCI) (19). The TCI assesses 4 temperament dimensions-NS, harm avoidance, reward dependence, and persistence — in addition to several subdimensions of NS (exploratory excitability, impulsiveness, extravagance, and disorderliness), harm avoidance (anticipatory worry, fear of uncertainty, shyness, and fatigability), and reward dependence (sentimentality, attachment, and dependence). The temperament dimensions of the Dutch version of the TCI have reasonable to good psychometric internal consistency (Cronbach's $\alpha$ range, 0.64-0.87) and include a validation in a reference sample $(n=1034)$ (version 1.3; Datec Psychologic Tests).

\section{mGluR5 PET Imaging}

${ }^{18} \mathrm{~F}$-FPEB was synthetized on-site using the nitro-precursor obtained from $\mathrm{ABX}$ (Advanced Biochemical Compounds). The time interval between the screening and PET sessions was $22 \pm 23 \mathrm{~d}$ (range, 2-78 d). A detailed optimized version of the existing labeling method (25) will be reported elsewhere. Before imaging, subjects were asked to fast for $3 \mathrm{~h}$ and to abstain from caffeine and alcohol consumption for at least 12 and $24 \mathrm{~h}$, respectively, and urine drug tests were repeated to ensure negative results. A venous and a lower radial arterial catheter were placed in opposite arms for tracer injection and radiometabolite-corrected input function determination. The method used to obtain the radiometabolitecorrected input function has been described previously (21). Tracer parent fractions in plasma were fitted with a sigmoid function. PET data were acquired on a Biograph $16 \mathrm{HiRez}$ camera (Siemens Inc.). An attenuation map was obtained by a low-dose ( $80 \mathrm{kV}, 13 \mathrm{~mA} \cdot \mathrm{s}) \mathrm{CT}$ scan. Dynamic PET acquisition was performed for 90 min after bolus injection of ${ }^{18} \mathrm{~F}$-FPEB (mean \pm SD injected activity, specific activity, and mass were $176.75 \pm 6.91 \mathrm{MBq}, 89.36 \pm 49.38 \mathrm{MBq} / \mathrm{nmol}$, and $0.53 \pm 0.04 \mu \mathrm{g}$, respectively).

PET data were analyzed using PMOD software (version 3.605; PMOD Technologies). Regional mGluR5 availability was quantified as the ${ }^{18} \mathrm{~F}$ FPEB total distribution volume $\left(\mathrm{V}_{\mathrm{T}}\right)$. To allow efficient voxel-based parametric mapping of $\mathrm{V}_{\mathrm{T}}$ calculation, the Logan graphical approach was used (21). For subsequent statistical parametric mapping (SPM) analysis, $\mathrm{V}_{\mathrm{T}}$ parametric images were spatially normalized to the Montreal Neurologic Institute space. Additionally, to corroborate voxel-based findings, a volume-of-interest (VOI) analysis was performed using the N30R83 Hammers probabilistic atlas (26), allowing for an observer-independent delineation of neuroanatomical regions. VOI-based $\mathrm{V}_{\mathrm{T}}$ values were derived from a reversible 2-tissue-compartment model $(18,21)$, with and without application of partial-volume effect correction. The geometric transfer matrix method described by Rosset et al. (27) was used for partial-volume correction.

\section{MR Data Acquisition and Processing}

MR structural imaging and spectroscopy were obtained in a single session on a 3-T scanner (Ingenia; Philips). A standard brain T1-weighted magnetization-prepared rapid gradient-echo sequence (repetition/echo time, 9.6/4.6 ms; flip angle, $8^{\circ}$; voxel size, $0.98 \times 0.98 \times 1.2 \mathrm{~mm}$ ) was acquired for VOs segmentation and coregistration with PET images. Subsequently, absolute ACC glutamate-glutamine concentrations were derived from single-voxel proton MR spectroscopy (MRS) using a point-resolved spectroscopy pulse sequence (repetition/echo time, 2,000/23 ms; receiver bandwidth, $2 \mathrm{kHz} ; 128$ averages; vector size, 1,024). A $15 \times 15 \times 15 \mathrm{~mm}$ $\left(3.4 \mathrm{~cm}^{3}\right)$ MRS voxel was placed in the bilateral pregenual ACC (Supplemental Fig. 1; supplemental materials are available at http://jnm. snmjournals.org). Proton MRS spectra were analyzed using jMRUI software (http://www.mrui.uab.es/mrui) (28). Data are reported as a ratio to unsuppressed water signal.

\section{Statistical Analysis}

Conventional statistical analyses were performed using Statistica (version 12; Statsoft). Shapiro-Wilk tests were used to test the distribution of variables against normality. Normally distributed data are reported as mean \pm $\mathrm{SD}$ and skewed data as median (interquartile range). TCI scores were transformed to $z$ scores based on the reference sample $(n=1,034)$. Because mGluR5 is suggested to be involved in normal aging (29) and influenced by estrogen signaling (30), age and sex were included as nuisance variables in all analyses. PET data were primarily analyzed on a voxel-by-voxel basis using SPM12 (http://www.fil.ion.ucl.ac.uk/spm/software/spm12/). Beforehand, $\mathrm{V}_{\mathrm{T}}$ parametric images were spatially smoothed using a gaussian kernel of $12 \times 12 \times 12 \mathrm{~mm}$ in full width at half maximum. Four multiple regression analyses were performed, one for each independent TCItemperament main scale. Voxels with a value below $80 \%$ of the global value were discarded. The statistical threshold was set at a $P$ value of less than 0.05 , corrected for familywise error, with a minimal cluster size of 200 voxels $\left(>1.6 \mathrm{~cm}^{3}\right)$. Significant clusters were anatomically labeled and visualized using MRICron (http://www.mccauslandcenter.sc. edu/mricro/mricron) and xjView toolbox (http://www.alivelearn.net/xjview). To minimize the chance of type I error, correlations with temperament 
subdimensions were performed only when positive findings were obtained for the main dimension. Similarly, in the secondary VOI-based analysis, partial Pearson correlations were assessed only for VOIs corresponding to brain regions with significant SPM results. To further ensure the robustness of data to age affects, post hoc analyses using data from volunteers $30 \mathrm{y}$ or younger and $40 \mathrm{y}$ or older were performed.

\section{RESULTS}

\section{Participants}

The mean age, body mass index, and years of education of the study sample ( $n=44 ; 22$ women) were $40 \pm 14 \mathrm{y}, 24 \pm 4 \mathrm{~kg} / \mathrm{m}^{2}$, and $16 \pm 3 \mathrm{y}$, respectively. Four subjects were left-handed, and 40 were right-handed. The median reported alcohol consumption was 1.3 (2.8) units/wk, and the average Alcohol Use Disorder Identification Test score was $2.2 \pm 1.6$.

\section{TCI Scores}

TCI temperament raw scores and $z$ scores are presented in Supplemental Table 1. After Bonferroni adjustment (4 temperament main dimensions), there were no differences between the study and reference sample, nor between male and female participants. This supports the absence of temperament dimensions inclusion bias. Regarding age, only the NS subdimension exploratory excitability significantly decreased with age $(r=-0.35 ; P=0.02)$, but this did not survive Bonferroni adjustment. We observed no significant intercorrelations between the main dimension scores. All subdimensions correlated positively with their respective main dimension (all $r>0.38 ; P<0.01$ ).

\section{Brain mGluR5 Availability}

Arterial radiometabolite fraction results are shown in Supplemental Figure 2. The regional brain mGluR5 availability of the study sample was in agreement with the known distribution of mGluR5 in humans $(14,31)$, with highest availability in the ACC, insula, and prefrontal regions and lowest availability in cerebellum and pons (Supplemental Fig. 3). There were no significant sex differences in both voxel- and VOI-based regional binding. We observed a significant decrease in mGluR5 availability with age in most brain VOIs, which remained significant after partial-volume effect correction. After Bonferroni adjustment ( 83 VOIs tested; $P<0.0006$ ), this negative association with age remained significant bilaterally in the post- and precentral gyri ( $r=-0.53$ and -0.51 , respectively), cuneus $(r=-0.49)$, and inferiolateral temporal lobe $(r=-0.51)$.

\section{Association Between mGluR5 Availability and Temperaments}

The voxel-based analysis showed a positive correlation between the trait NS and mGluR5 availability in several brain clusters (Table 1; Fig. 1), including mostly symmetric paralimbic and primary

TABLE 1

SPM of Positive Correlation Between mGluR5 Availability and Temperament Trait NS

\begin{tabular}{|c|c|c|c|c|c|c|c|c|c|}
\hline \multicolumn{2}{|c|}{ Cluster level } & \multicolumn{2}{|c|}{ Voxel level } & \multicolumn{3}{|c|}{$\begin{array}{l}\text { Peak voxel MNI } \\
\text { coordinate }\end{array}$} & \multicolumn{3}{|l|}{ Location } \\
\hline $\mathrm{K}_{\mathrm{E}}$ & $P^{*}$ & t score & $P^{*}$ & $x$ & $y$ & $z$ & Anatomic area ${ }^{\dagger}$ & Hemisphere & $\mathrm{BA}$ \\
\hline \multirow[t]{4}{*}{7719} & $<0.001$ & 6.07 & $<0.001$ & 17 & -28 & 5 & Thalamus (pulvinar) & Right & - \\
\hline & & 5.71 & 0.001 & -13 & -30 & 3 & Thalamus (pulvinar) & Left & - \\
\hline & & 5.04 & 0.005 & -25 & -28 & -19 & Parahippocampal and ambient gyri & Left & 35 \\
\hline & & 4.92 & 0.007 & -17 & -8 & -23 & Amygdala & Left & 34 \\
\hline \multirow[t]{3}{*}{1807} & 0.002 & 4.65 & 0.01 & 45 & -4 & -9 & Insula & Right & 21 \\
\hline & & 4.53 & 0.02 & 43 & -20 & 9 & Superior temporal gyrus, posterior part & Right & 13 \\
\hline & & 4.49 & 0.02 & 41 & 18 & -31 & Superior temporal gyrus, anterior part & Right & 38 \\
\hline \multirow[t]{3}{*}{748} & 0.009 & 4.61 & 0.02 & -15 & -86 & 15 & Lateral remainder of occipital lobe & Left & 18 \\
\hline & & 4.51 & 0.02 & 7 & -76 & 7 & Cuneus & Right & 17 \\
\hline & & 4.25 & 0.04 & 13 & -84 & 21 & Cuneus & Right & 18 \\
\hline \multirow[t]{2}{*}{231} & 0.02 & 4.52 & 0.02 & 57 & -14 & 43 & Postcentral gyrus & Right & 3 \\
\hline & & 4.27 & 0.04 & 37 & -32 & 59 & Postcentral gyrus & Right & 3 \\
\hline 177 & 0.03 & 4.44 & 0.03 & -1 & -12 & 43 & Posterior cingulate gyrus & Left & 24 \\
\hline \multirow[t]{2}{*}{271} & 0.02 & 4.39 & 0.03 & -41 & -30 & 52 & Postcentral gyrus & Left & 3 \\
\hline & & 4.28 & 0.04 & -39 & -16 & 52 & Precentral gyrus & Left & 4 \\
\hline 120 & 0.03 & 4.38 & 0.03 & 19 & 0 & -21 & Amygdala & Right & 34 \\
\hline \multirow[t]{2}{*}{218} & 0.02 & 4.37 & 0.03 & 11 & -52 & 37 & Superior parietal gyrus (precuneus) & Right & 31 \\
\hline & & 4.21 & 0.04 & -1 & -42 & 35 & Posterior cingulate gyrus & Left & 31 \\
\hline \multirow[t]{2}{*}{204} & 0.03 & 4.32 & 0.04 & -3 & 42 & 0 & Anterior cingulate gyrus & Left & 32 \\
\hline & & 4.30 & 0.04 & 1 & 36 & 16 & Anterior cingulate gyrus & Right & 32 \\
\hline
\end{tabular}

${ }^{*}$ Corrected for familywise error.

${ }^{\dagger}$ According to Hammers et al. (26).

$\mathrm{BA}=$ Brodmann area; $\mathrm{K}_{\mathrm{E}}=$ cluster size extent (number of $2 \times 2 \times 2 \mathrm{~mm}$ voxels); $\mathrm{MNI}=$ Montreal Neurologic Institute. 


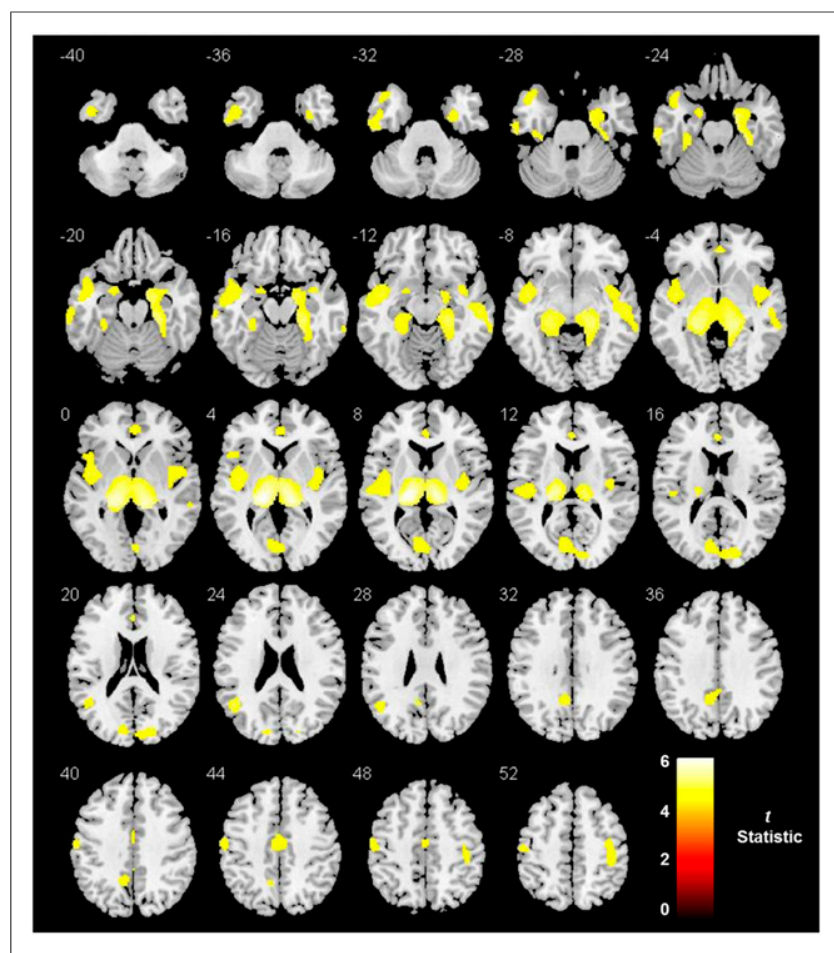

FIGURE 1. Positive correlation between cerebral mGluR5 availability $\left({ }^{18} \mathrm{~F}-\mathrm{FPEB} \mathrm{V}_{\mathrm{T}}\right)$ and temperament trait NS. SPM displayed at $\mathrm{P}$ height of $P<0.05$ (corrected for familywise error) and cluster size extent greater than 200 voxels $\left(>1.6 \mathrm{~cm}^{3}\right)$. Clusters were overlaid onto canonical Montreal Neurologic Institute brain template (axial sections in radiologic orientation, $z$-coordinate indicated). sensory areas. The strongest correlations were found in a large cluster including the bilateral thalamus (peak location in the pulvinar) and medial structures of the left temporal lobe. Figure 2 shows most paralimbic clusters along with regression analyses at local maxima. There were no significant negative associations between regional mGluR5 availability and NS or any significant correlations between mGluR5 and other temperament traits.

Table 2 shows the results of the VOI-based analysis, complementing voxel-based findings. VOI-based data largely confirm the positive relationship seen in SPM, with some discrepancies regarding the location of highest associations probably reflecting the fact that VOI-based data do not inform about peak value locations at the voxel level. Partial-volume effects correction did not affect findings (Supplemental Table 2), supporting that gray matter volume differences did not influence results. Within the NS dimension, mGluR5 availability was mainly correlated to the subdimension exploratory excitability (Supplemental Table 3).

To further substantiate the specificity of the results, we performed the same analyses on the character dimensions of the TCI, which revealed no significant mGluR5 associations with any of the scales. Also, main findings remained when data from volunteers $30 \mathrm{y}$ or younger or $40 \mathrm{y}$ or older were used, indicating the robustness of data to age effects (Supplemental Tables 4 and 5).

\section{MRS}

Of the 44 MRS spectra, 2 were of too-low quality to allow reliable quantification and were excluded from the analysis. The mean glutamate and glutamine concentrations in the subgenual ACC were $12.82 \pm 2.36$ and $1.57 \pm 0.90 \mathrm{mmol} / \mathrm{L}$, respectively ( $n=42)$, consistent with previous reports in healthy humans (32). Here, MRS-derived ACC glutamate and glutamine (or the combination of both, that is, Glx) concentrations did not correlate significantly with personality measures or with regional mGluR5 availability (all $P>0.15)$.

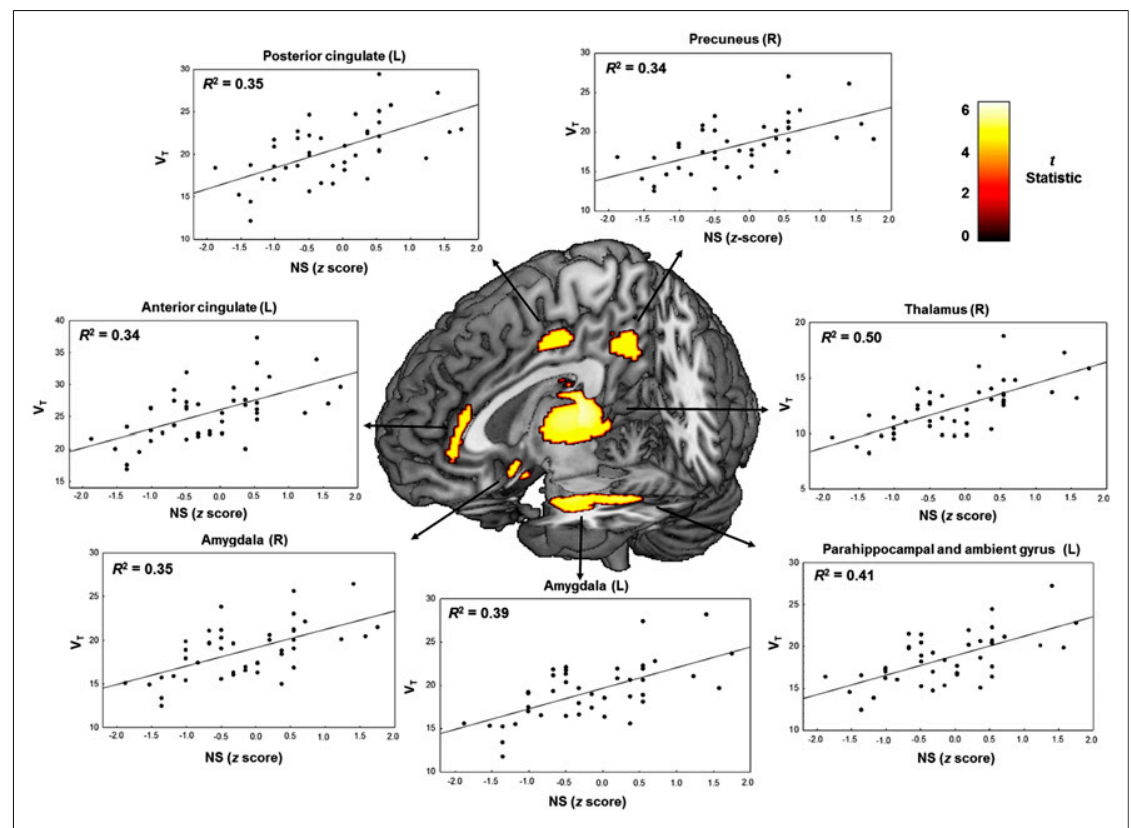

FIGURE 2. Positive correlation between mGluR5 availability $\left({ }^{18} \mathrm{~F}-\mathrm{FPEB} \mathrm{V}_{\mathrm{T}}\right)$ in limbic areas and temperament trait NS. SPM displayed at $\mathrm{P}$ height of $P<0.05$ (corrected for familywise error) and cluster size extent greater than 200 voxels $\left(>1.6 \mathrm{~cm}^{3}\right)$. Clusters were overlaid onto canonical 3dimensional-rendered MRI brain template with a cut-out. Linear regression analysis using extracted $\mathrm{V}_{\mathrm{T}}$ values at local maxima is given for each regional cluster (arrows).

\section{DISCUSSION}

Combining molecular imaging with personality assessments in healthy human adults, we found a strong positive and specific association between the temperament trait NS and mGluR5 availability in limbic and paralimbic areas, as well as in various primary sensory cortices.

NS is a multifaceted construct that reflects a bias toward intense excitement in response to novelty, leading to frequent engagement in novel and highly emotional experiences, and extravagance in response to signals of reward $(1,19)$. Individuals scoring high in NS are excitable, quick-tempered, and impulsive. Heritability of NS behavior has been estimated to be $41 \%$ (33). Excessive NS has been consistently linked to several neuropsychiatric disorders, including substance abuse (4), pathologic gambling (34), and eating disorders (35). Although the role of dopamine in driving NS behaviors has been largely confirmed (36-38), the involvement of other neurotransmitter systems remains scarcely studied. 
TABLE 2

Partial Correlation Coefficients* Between mGluR5 Availability and Temperament Traits

\begin{tabular}{|c|c|c|c|c|c|c|c|c|c|}
\hline \multirow[b]{2}{*}{ Volume of interest ${ }^{\dagger}$} & \multirow[b]{2}{*}{ Hemisphere } & \multicolumn{2}{|c|}{$\begin{array}{l}\text { Novelty } \\
\text { seeking }\end{array}$} & \multicolumn{2}{|c|}{$\begin{array}{c}\text { Harm } \\
\text { avoidance }\end{array}$} & \multicolumn{2}{|c|}{$\begin{array}{c}\text { Reward } \\
\text { dependence }\end{array}$} & \multicolumn{2}{|c|}{ Persistence } \\
\hline & & $r$ & $P$ & $r$ & $P$ & $r$ & $P$ & $r$ & $P$ \\
\hline \multirow[t]{2}{*}{ Thalamus } & Right & 0.59 & $<0.001$ & 0.00 & 0.984 & 0.17 & 0.291 & -0.07 & 0.650 \\
\hline & Left & 0.61 & $<0.001$ & -0.01 & 0.942 & 0.25 & 0.116 & -0.18 & 0.252 \\
\hline \multirow[t]{2}{*}{ Parahippocampal and ambient gyrus } & Right & 0.66 & $<0.001$ & 0.07 & 0.681 & 0.17 & 0.277 & -0.18 & 0.262 \\
\hline & Left & 0.53 & $<0.001$ & -0.01 & 0.932 & 0.17 & 0.280 & -0.09 & 0.564 \\
\hline \multirow[t]{2}{*}{ Amygdala } & Right & 0.63 & $<0.001$ & -0.02 & 0.911 & 0.31 & 0.049 & -0.15 & 0.358 \\
\hline & Left & 0.57 & $<0.001$ & -0.02 & 0.882 & 0.22 & 0.163 & -0.16 & 0.306 \\
\hline \multirow[t]{2}{*}{ Insula } & Right & 0.60 & $<0.001$ & -0.01 & 0.957 & 0.21 & 0.174 & -0.12 & 0.436 \\
\hline & Left & 0.58 & $<0.001$ & -0.08 & 0.615 & 0.18 & 0.256 & -0.15 & 0.329 \\
\hline \multirow[t]{2}{*}{ Anterior cingulate cortex } & Right & 0.53 & $<0.001$ & 0.09 & 0.582 & 0.08 & 0.608 & -0.21 & 0.183 \\
\hline & Left & 0.55 & $<0.001$ & -0.06 & 0.687 & 0.23 & 0.146 & -0.14 & 0.360 \\
\hline \multirow[t]{2}{*}{ Posterior cingulate cortex } & Right & 0.59 & $<0.001$ & -0.09 & 0.550 & 0.18 & 0.247 & -0.14 & 0.381 \\
\hline & Left & 0.57 & $<0.001$ & -0.01 & 0.932 & 0.15 & 0.328 & -0.17 & 0.294 \\
\hline \multirow[t]{2}{*}{ Superior parietal gyrus } & Right & 0.60 & $<0.001$ & -0.01 & 0.940 & 0.16 & 0.303 & -0.12 & 0.452 \\
\hline & Left & 0.57 & $<0.001$ & 0.02 & 0.879 & 0.16 & 0.298 & -0.15 & 0.352 \\
\hline \multirow[t]{2}{*}{ Cuneus } & Right & 0.59 & $<0.001$ & -0.08 & 0.614 & 0.25 & 0.113 & -0.09 & 0.588 \\
\hline & Left & 0.51 & $<0.001$ & 0.05 & 0.714 & 0.17 & 0.269 & -0.15 & 0.334 \\
\hline
\end{tabular}

${ }^{*}$ Controlling for age and sex.

${ }^{\dagger}$ According to Hammers et al. (26).

The present findings suggest that regional mGluR5 availability, with the thalamus as a core component, is a major modulator of NS behaviors in humans. A first critical facet of NS is perceptual, because the response to novel and reward sensory stimuli initially triggers the behavior. The topography of mGluR5/NS associations includes first-order thalamic nuclei (lateral geniculate, medial geniculate, and ventral posterior nuclei), representing the first relay of visual, auditory, and somatosensory information to their respective primary cortex. Remarkably, we also observed mGluR5temperament associations in these primary sensory cortices. These data suggest that mGluR5 may modulate NS at the earliest processing stages of sensory stimuli, which is in strong agreement with Cloninger's view of temperament, that is, preconceptual bias in perceptual memory and habit formation. A second critical aspect of NS is emotional, because the urge toward vigorous emotional sensations and quick loss of temper are key features of high NS. The extensive paralimbic associations observed strongly support a role for mGluR5 in this emotional facet of NS. Mounting evidence indicates that the thalamus actively modulates cortical and subcortical responses, aside from passively relaying information to the cortex. Here, the strongest associations were found in the limbic thalamus, including the pulvinar and midline nuclei (39). These nuclei have dense connections with other paralimbic structures and are critically involved in inappropriate action inhibition and behaviors such as drug seeking and feeding (40). Previously, an association between the cannabinoid receptor subtype 1 in the human amygdala and NS has been reported (6). Because mGluR5 interacts with presynaptic cannabinoid receptor subtype 1 to regulate top-down cognitive control (13), limbic mGluR5/cannabinoid signaling interactions could partially ac- count for individual differences in NS behavior. In addition to sensory and emotional aspects, a third critical facet of NS is reward. Here, we did not observe associations between mGluR5 and NS in core regions of the reward circuitry, that is, the ventral tegmental area and the ventral striatum. Though this aspect of NS might primarily be related to dopamine signaling in humans (36), a recent study found that specific inactivation of mGluR5 signaling in D1-dopaminoceptive neurons abolishes NS behaviors in rodents (16), still suggesting that some dopaminergic-glutamatergic interactions are involved in NS behaviors. Lastly, the insula is a paralimbic region critically involved in determining the emotionalmotivational salience of various somatosensory stimuli (41), a key aspect of NS. High NS personality has been associated with increased metabolic activity in the right insula (42), and the role of the insula in relating risk prediction to NS behavior has been documented (43). Given that mGluR5 modulates synaptic transmission, a glutamatergic basis of these associations can be hypothesized.

Here, we found a negative association between mGluR5 availability and age in most brain regions, supporting the proposed involvement of this receptor in normal aging (29). This contrasts with the absence of age dependency recently reported using ${ }^{11} \mathrm{C}$ ABP688, another mGluR5 tracer (31). This discrepancy could be explained by the higher affinity and signal-to-noise ratio of ${ }^{18} \mathrm{~F}$ FPEB for mGluR5 (18). Interestingly, the present findings were mostly driven by the NS subdimension exploratory excitability (a concept close to sensation seeking), which also showed a negative association with age. As our inclination toward thrill-seeking behaviors is higher during young adulthood than at older ages, it is tempting to suggest a role for mGluR5 in this observation. 
Finally, we found no relationship between MRS-proxy measures of glutamate transmission in the ACC and temperaments. This contrasts with previous studies in which ACC glutamate levels have been associated positively with sensation seeking (8) and negatively with harm avoidance (9). Possible explanations for these discrepancies include for the former a difference in MRSVOI location (pregenual vs. dorsal ACC) and in the behavior construct measured (novelty vs. sensation seeking), and for both studies a difference in data processing.

Some limitations to the present study should be mentioned. First, the study sample size was relatively small, though large enough to draw robust conclusions. Second, because of the absence of a reference region devoid of mGluR5 in the human brain (14), direct estimation of specific radiotracer binding (i.e., the nondisplaceable binding potential) is not possible. Indirect nondisplaceable binding potential estimation from kinetic modeling is challenging and must be validated by earlier blocking studies, which are lacking for ${ }^{18} \mathrm{~F}-$ FPEB. Therefore, $V_{T}$, including both specific and nonspecific binding, was used. $V_{T}$ values were consistent with the known distribution of mGluR5 in humans, and the reliability of this parameter has been demonstrated $(18,21)$, supporting its validity to quantify mGluR5 availability. Third, as suggested for another allosteric mGluR5 PET tracer (20), variation in extracellular glutamate levels could have affected findings. Here, we found no significant correlations between ACC glutamate/glutamine levels and tracer binding. However, this does not exclude that mGluR5 availability might be influenced by glutamate levels in other brain areas or by larger changes in metabolite concentrations. Fourth, given the known effect of age on both NS and cortical atrophy, age might have confounded our results. To prevent this possibility, age was used as nuisance variable in all analyses. Moreover, results were confirmed using both partial-volume effect-corrected PET values and post hoc analyses with data from volunteers $30 \mathrm{y}$ or younger or $40 \mathrm{y}$ or older, supporting the robustness of data to age effects. Also, given the influence of estrogens on mGluR5 and dopamine signaling, a subgroup analysis based on menopausal status would have been informative but was not performed because only 5 women entered menopause at inclusion time. Finally, given the heritable component of NS, the effect of mGluR5 polymorphisms on mGluR5 availability would be interesting to address in future studies.

\section{CONCLUSION}

We found that high NS temperament is robustly associated with high mGluR5 availability in key limbic and primary sensory brain areas, with thalamic mGluR5 explaining up to $50 \%$ of the NS variance in the sample. These findings add to our understanding of the complex neurobiologic correlates of human personality by suggesting, beyond the established role of various monoamine systems, involvement of the modulatory glutamatergic system. Intensified investigation of the link between mGluR5 and NS is strongly supported, especially in light of the known role of mGluR5 in addiction and other disorders associated with high NS temperament.

\section{DISCLOSURE}

The costs of publication of this article were defrayed in part by the payment of page charges. Therefore, and solely to indicate this fact, this article is hereby marked "advertisement" in accordance with 18 USC section 1734. Financial support was received from the Flemish Fund for Scientific Research, Flanders, Belgium
(FWO/G.0548.06). Koen Van Laere is a senior clinical investigator for the FWO. No other potential conflict of interest relevant to this article was reported.

\section{ACKNOWLEDGMENTS}

We acknowledge the contribution of Kwinten Porters, the radiopharmacy and radiology team UZ Leuven, and Prof. Dr. Hugo Neels, the Toxicological Center of the Antwerp University, and the Toxicology and TDM Laboratory of the ZNA Stuivenberg Hospital for the performed ethyl glucuronide hair analyses.

\section{REFERENCES}

1. Cloninger CR. A systematic method for clinical description and classification of personality variants: a proposal. Arch Gen Psychiatry. 1987;44:573-588.

2. Ebstein RP. The molecular genetic architecture of human personality: beyond self-report questionnaires. Mol Psychiatry. 2006;11:427-445.

3. Rettew DC, McKee L. Temperament and its role in developmental psychopathology. Harv Rev Psychiatry. 2005;13:14-27.

4. Bidwell LC, Knopik VS, Audrain-McGovern J, et al. Novelty seeking as a phenotypic marker of adolescent substance use. Subst Abuse. 2015;9:1-10.

5. Schreckenberger M, Klega A, Grunder G, et al. Opioid receptor PET reveals the psychobiologic correlates of reward processing. J Nucl Med. 2008;49:1257-1261.

6. Van Laere K, Goffin K, Bormans G, et al. Relationship of type 1 cannabinoid receptor availability in the human brain to novelty-seeking temperament. Arch Gen Psychiatry. 2009;66:196-204.

7. Ende G, Cackowski S, Van Eijk J, et al. Impulsivity and aggression in female BPD and ADHD patients: association with ACC glutamate and GABA concentrations. Neuropsychopharmacology. 2016;41:410-418.

8. Gallinat J, Kunz D, Lang UE, et al. Association between cerebral glutamate and human behaviour: the sensation seeking personality trait. Neuroimage. 2007;34: 671-678.

9. Kim HJ, Kim JE, Cho G, et al. Associations between anterior cingulate cortex glutamate and gamma-aminobutyric acid concentrations and the harm avoidance temperament. Neurosci Lett. 2009;464:103-107.

10. Gleich T, Lorenz RC, Pohland L, et al. Frontal glutamate and reward processing in adolescence and adulthood. Brain Struct Funct. 2015;220:3087-3099.

11. Modi S, Rana P, Kaur P, Rani N, Khushu S. Glutamate level in anterior cingulate predicts anxiety in healthy humans: a magnetic resonance spectroscopy study. Psychiatry Res. 2014;224:34-41.

12. Benquet $P$, Gee $\mathrm{CE}$, Gerber U. Two distinct signaling pathways upregulate NMDA receptor responses via two distinct metabotropic glutamate receptor subtypes. J Neurosci. 2002;22:9679-9686.

13. Kiritoshi T, Sun H, Ren W, et al. Modulation of pyramidal cell output in the medial prefrontal cortex by mGluR5 interacting with CB1. Neuropharmacology. 2013;66:170-178.

14. Patel S, Hamill TG, Connolly B, Jagoda E, Li W, Gibson RE. Species differences in mGluR5 binding sites in mammalian central nervous system determined using in vitro binding with [ ${ }^{18}$ F]F-PEB. Nucl Med Biol. 2007;34:1009-1017.

15. Ballard TM, Woolley ML, Prinssen E, Huwyler J, Porter R, Spooren W. The effect of the mGlu5 receptor antagonist MPEP in rodent tests of anxiety and cognition: a comparison. Psychopharmacology (Berl). 2005;179:218-229.

16. Parkitna JR, Sikora M, Golda S, et al. Novelty-seeking behaviors and the escalation of alcohol drinking after abstinence in mice are controlled by metabotropic glutamate receptor 5 on neurons expressing dopamine d1 receptors. Biol Psychiatry. 2013;73:263-270.

17. Chiamulera C, Epping-Jordan MP, Zocchi A, et al. Reinforcing and locomotor stimulant effects of cocaine are absent in mGluR5 null mutant mice. Nat Neurosci. 2001;4:873-874.

18. Wong DF, Waterhouse R, Kuwabara H, et al. ${ }^{18}$ F-FPEB, a PET radiopharmaceutical for quantifying metabotropic glutamate 5 receptors: a first-in-human study of radiochemical safety, biokinetics, and radiation dosimetry. J Nucl Med. 2013; 54:388-396.

19. Cloninger CR, Svrakic DM, Przybeck TR. A psychobiological model of temperament and character. Arch Gen Psychiatry. 1993;50:975-990.

20. DeLorenzo C, DellaGioia N, Bloch M, et al. In vivo ketamine-induced changes in $\left[{ }^{11} \mathrm{C}\right] \mathrm{ABP} 688$ binding to metabotropic glutamate receptor subtype 5. Biol Psychiatry. 2015;77:266-275.

21. Leurquin-Sterk G, Postnov A, de Laat B, et al. Kinetic modeling and long-term test-retest reproducibility of the mGluR5 PET tracer ${ }^{18} \mathrm{~F}-\mathrm{FPEB}$ in human brain. Synapse. 2016;70:153-162. 
22. Sheehan DV, Lecrubier Y, Sheehan KH, et al. The mini-international neuropsychiatric interview (M.I.N.I.): the development and validation of a structured diagnostic psychiatric interview for DSM-IV and ICD-10. J Clin Psychiatry. 1998;59(suppl 20):22-33.

23. Saunders JB, Aasland OG, Babor TF, de la Fuente JR, Grant M. Development of the alcohol use disorders identification test (AUDIT): WHO collaborative project on early detection of persons with harmful alcohol consumption-II. Addiction. 1993;88:791-804.

24. de la Rie SM, Duijsens IJ, Cloninger CR. Temperament, character, and personality disorders. J Pers Disord. 1998;12:362-372.

25. Liang SH, Yokell DL, Jackson RN, et al. Microfluidic continuous-flow radiosynthesis of $\left[{ }^{18} \mathrm{~F}\right] \mathrm{FPEB}$ suitable for human PET imaging. MedChemComm. 2014; 5:432-435.

26. Hammers A, Allom R, Koepp MJ, et al. Three-dimensional maximum probability atlas of the human brain, with particular reference to the temporal lobe. Hum Brain Mapp. 2003;19:224-247.

27. Rousset OG, Ma Y, Evans AC. Correction for partial volume effects in PET: principle and validation. J Nucl Med. 1998;39:904-911.

28. Naressi A, Couturier C, Devos JM, et al. Java-based graphical user interface for the MRUI quantitation package. MAGMA. 2001;12:141-152.

29. Ménard C, Quirion R. Successful cognitive aging in rats: a role for mGluR5 glutamate receptors, homer 1 proteins and downstream signaling pathways. PLoS One. 2012;7:e28666.

30. Grove-Strawser D, Boulware MI, Mermelstein PG. Membrane estrogen receptors activate the metabotropic glutamate receptors mGluR5 and mGluR3 to bidirectionally regulate CREB phosphorylation in female rat striatal neurons. Neuroscience. 2010;170:1045-1055.

31. DuBois JM, Rousset OG, Rowley J, et al. Characterization of age/sex and the regional distribution of mGluR5 availability in the healthy human brain measured by high-resolution $\left[{ }^{11} \mathrm{C}\right] \mathrm{ABP} 688$ PET. Eur J Nucl Med Mol Imaging. 2016;43:152-162.
32. Pouwels PJ, Frahm J. Regional metabolite concentrations in human brain as determined by quantitative localized proton MRS. Magn Reson Med. 1998;39: 53-60.

33. Heath AC, Cloninger CR, Martin NG. Testing a model for the genetic structure of personality: a comparison of the personality systems of Cloninger and Eysenck. J Pers Soc Psychol. 1994;66:762-775.

34. Kim SW, Grant JE. Personality dimensions in pathological gambling disorder and obsessive-compulsive disorder. Psychiatry Res. 2001;104:205-212.

35. Krug I, Pinheiro AP, Bulik C, et al. Lifetime substance abuse, family history of alcohol abuse/dependence and novelty seeking in eating disorders: comparison study of eating disorder subgroups. Psychiatry Clin Neurosci. 2009;63:82-87.

36. Zald DH, Cowan RL, Riccardi P, et al. Midbrain dopamine receptor availability is inversely associated with novelty-seeking traits in humans. J Neurosci. 2008; 28:14372-14378.

37. Kaasinen V, Aalto S, Nagren K, Rinne JO. Insular dopamine D2 receptors and novelty seeking personality in Parkinson's disease. Mov Disord. 2004;19:13481351.

38. Laine TP, Ahonen A, Rasanen P, Tiihonen J. Dopamine transporter density and novelty seeking among alcoholics. J Addict Dis. 2001;20:91-96.

39. Taber KH, Wen C, Khan A, Hurley RA. The limbic thalamus. J Neuropsychiatry Clin Neurosci. 2004;16:127-132.

40. Saalmann YB, Kastner S. The cognitive thalamus. Front Syst Neurosci. 2015; 9:1-2.

41. Seeley WW, Menon V, Schatzberg AF, et al. Dissociable intrinsic connectivity networks for salience processing and executive control. J Neurosci. 2007;27: 2349-2356.

42. Sugiura M, Kawashima R, Nakagawa M, et al. Correlation between human personality and neural activity in cerebral cortex. Neuroimage. 2000;11:541-546.

43. Wang Y, Liu Y, Yang L, et al. Novelty seeking is related to individual risk preference and brain activation associated with risk prediction during decision making. Sci Rep. 2015;5:10534. 